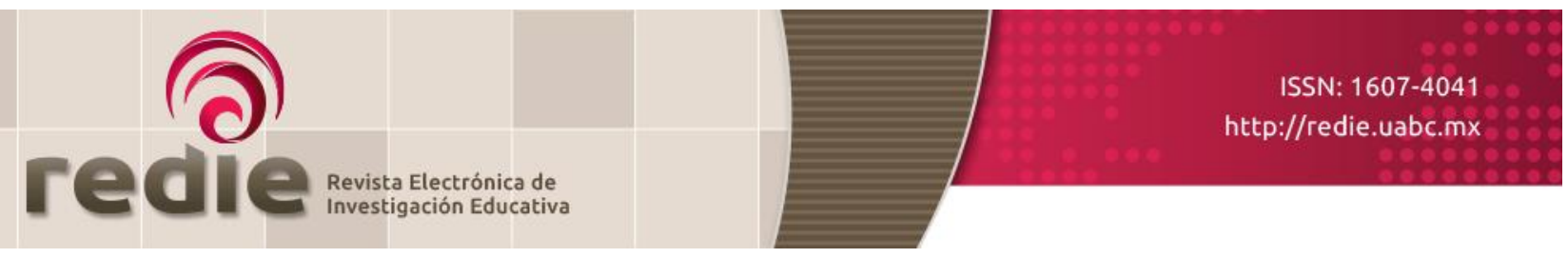

Vol. 20, Núm. 4, 2018

\title{
Clima motivacional percibido hacia el deporte y factores académicos en estudiantes universitarios de Granada
}

\section{Perceived Motivational Climate in Sport and Academic Factors in University Students from Granada}

\author{
Ramón Chacón Cuberos (*) ramonchaconcuberos@correo.ugr.es \\ Asunción Martínez Martínez (*) asuncionmm@ugr.es \\ Tamara Espejo Garcés (*) tamaraeg@correo.ugr.es \\ Manuel Castro Sánchez (*) manue87@correo.ugr.es \\ Félix Zurita Ortega (*) felixzo@ugr.es \\ Antonio José Pérez Cortés (*) antperez@ugr.es \\ (*) Universidad de Granada \\ (Recibido: 21de enero de 2017; Aceptado para su publicación: 11 de abril de 2017)
}

Cómo citar: Chacón, R., Martínez, A., Espejo, T., Castro, M., Zurita, F. y Pérez, A. J. (2018). Clima motivacional percibido hacia el deporte y factores académicos en estudiantes universitarios de Granada. Revista Electrónica de Investigación Educativa, 20(4), 100109. https://doi.org/10.24320/redie.2018.20.4.1795

\section{Resumen}

Este estudio de tipo descriptivo y corte transversal, realizado en una muestra de 490 estudiantes universitarios, tiene como objetivo analizar las relaciones existentes entre diversos factores académicos como el rendimiento escolar y la concesión de beca y el clima motivacional percibido hacia el deporte, empleando como principales instrumentos el cuestionario PMCSQ-2 y una hoja de auto-registro. Los resultados mostraron que la concesión de beca y la vía de acceso a la universidad no se relacionaban con el clima motivacional hacia el deporte. Por el contrario, se pudo observar que aquellos estudiantes que estaban matriculados en centros concertados o privados eran los que más se esforzaban para mejorar en la práctica deportiva, persiguiendo el reconocimiento desigual. Asimismo, se obtuvo que los participantes con mayores calificaciones preferían tener un papel protagonista en la práctica deportiva.

Palabras clave: Universidad, rendimiento académico, motivación, deporte.

\section{Abstract}

This descriptive and cross-sectional study, conducted on a sample of 490 university students, seeks to analyze the relationships between academic factors such as academic achievement and holding a scholarship, and the perceived motivational climate in sport. The main instruments used were the PMCSQ-2 questionnaire and a self-registration sheet. The results showed that holding a scholarship and students' access route to university were not related to the motivational climate in sport. It was however observed that students enrolled in privately-run state-funded schools (centros concertados) or private 
schools strived hardest to improve in sports and sought recognition. Furthermore, it was found that participants with higher grades prefer to play a leading role in sports.

Keywords: University, academic performance, motivation, sport.

\section{Introducción}

La universidad constituye la cúspide del sistema educativo actual, pues es la institución encargada de desarrollar, transmitir y criticar la cultura y la ciencia, así como preparar para el ejercicio profesional y llevar a cabo la formación permanente (Martínez-Martínez, 2013; Renault, Cortada y Castro, 2014). Este organismo representa un papel central en la sociedad del conocimiento, ya que otorga los grados académicos profesionalizantes de los diversos campos del saber (Martínez-Martínez, 2013). En este sentido, el estudiante universitario se caracteriza por pertenecer al sector de adultos jóvenes que desempeña sus estudios en instituciones universitarias con el fin de obtener la formación necesaria que le habilite para ejercer una profesión especializada (Aránguiz y Rivera, 2012; Martínez, Castro, Zurita y Lucena, 2015).

El rendimiento académico, especialmente en el sector universitario, constituye uno de los principales elementos que pueden operar en la consecución de metas a nivel laboral y profesional (Camacho-Miñano y Del Campo, 2015). Autores como Agasisti y Ricca (2015) o Elias y Daza (2014) han puesto en relieve el papel de la tipología del centro universitario o la obtención de becas y proyectos como agentes modulares del éxito en la etapa universitaria. Asimismo, se ha demostrado cómo el estilo de vida y factores motivacionales ejercen una influencia directa en el logro académico. De hecho, Alfonso (2016) y Ayan, Besada, Cancelas y Martínez (2015) no sólo muestran una relación directa entre práctica deportiva y un mejor rendimiento académico mediante la eliminación de estados de ansiedad y estrés, sino también de forma indirecta; pues un estilo de vida activo se desvincula de conductas no saludables asociadas a bajo desempeño escolar, como el consumo de sustancias nocivas (Castro-Sánchez et al., 2015; Lisha y Sussman, 2010).

Resulta de interés estudiar algunos de los principales factores motivacionales que pueden influir en la práctica deportiva y el logro académico. La Teoría de la Autodeterminación (Deci y Ryan, 2000) se ha utilizado en las últimas décadas para dar explicación a los mecanismos implicados en la consecución de tareas de diversa índole (García-Calvo, Sánchez, Leo, Sánchez y Amado, 2011; Jaakkola, Ntoumanis y Liukkonen, 2016). Esta teoría establece que la motivación que interviene a la hora de realizar una acción configura un continuo, el cual varía en función del grado de autodeterminación del sujeto. En el extremo más autodeterminado del continuo se sitúa la motivación intrínseca y en la zona menos autodeterminada la desmotivación, situándose en la zona intermedia la motivación extrínseca. De este modo, autores como Marcos, Orquín, Belando y Moreno-Murcia (2014) recuerdan que la realización de ejercicio físico o práctica deportiva suele adherirse a un componente lúdico y hedonista, el cual se situaría en el extremo más autodeterminado, asociándose a motivaciones intrínsecas. Por otro lado, las tareas y acciones involucradas en el proceso de enseñanza-aprendizaje del mundo universitario se han relacionado más con motivaciones extrínsecas, como la obtención de oportunidades laborales (Camacho-Miñano y Del Campo, 2015; Martínez et al., 2015), aunque también puede existir un componente altruista.

En la práctica deportiva, uno de los modelos teóricos más utilizados en el desarrollo de los procesos motivacionales ha sido la Teoría de las Metas de Logro (Newton, Duda y Yin, 2000). Esta teoría se basa en las destrezas que posee cada sujeto, estableciendo que sus metas dependerán de la percepción de sus habilidades. De este modo, el clima motivacional podrá orientarse hacia la tarea o maestría, o bien hacia el ego o el rendimiento (Almagro, Sáenz-López y Moreno-Murcia, 2012; Cuevas, García-Calvo y Contreras, 2013; Méndez-Giménez, Fernández-Río y Cecchini-Estrada, 2014). En la dimensión Clima Tarea la motivación del sujeto se focaliza en el proceso a seguir, primando la mejora personal mediante el esfuerzo y el aprendizaje cooperativo, relacionándose con motivaciones intrínsecas. Por el contrario, en la dimensión Clima Ego las metas se orientan al rendimiento del individuo mediante un talento natural, el cual busca obtener mayor reconocimiento social que sus competidores (Almagro et al., 2015; Castillo, Balaguer y Duda, 2000; Méndez-Giménez, Fernández-Río, Cecchini-Estrada y González, 2013). 
Estudios recientes muestran la importancia de los procesos motivacionales en el rendimiento académico. Hanus y Fox (2015) revelan una relación directamente proporcional entre la motivación generada mediante técnicas de gamificación, el nivel de esfuerzo, satisfacción producida y logro académico. Asimismo, Kusurkar, Ten Cate, Vos, Westers y Croiset (2013) destacaron la relevancia de la autonomía y la motivación intrínseca en las buenas estrategias de estudio y las puntuaciones académicas más elevadas. En una línea similar, Ardoy et al. (2013) destacan cómo la práctica físico-deportiva favorece el rendimiento académico, demostrando que los adolescentes que practicaban actividad física con mayor asiduidad obtenían un mejor rendimiento cognitivo y escolar.

De este modo, y dada la influencia de diversos aspectos motivacionales y la práctica deportiva en el desempeño académico, este estudio persigue como principal objetivo describir las relaciones existentes entre el clima motivacional hacia el deporte y factores académicos concretos, como es la tipología del centro universitario, la concesión de cuantías económicas para estudiar o la nota de acceso a la universidad.

\section{Método}

\subsection{Diseño y participantes}

Este trabajo de investigación presenta un diseño no experimental, ex post-facto, de carácter descriptivo y correlacional mediante medición única. Asimismo, configura un modelo multifactorial univariado al definir el clima motivacional hacia el deporte como única variable dependiente. La muestra estuvo constituida por 490 estudiantes universitarios de Grado en Educación Primaria de la Universidad de Granada que cursaban "Enseñanza de la Educación Física en Educación Primaria". Se asumió un error muestral del 0.02 para 528 participantes de un universo total de 676 sujetos, aunque se eliminaron 38 cuestionarios por encontrarse mal cumplimentados. La edad de los participantes estuvo comprendida entre los 20 y 29 años ( $M=21.80$; $D T=3.63)$, representando un 39.4\% ( $n=193)$ a los hombres y un $60.6 \%(n=297)$ a las mujeres. Los centros que participaron en este trabajo de investigación fueron la Facultad de Ciencias de la Educación, el Centro de Magisterio "La Inmaculada" y la Escuela Universitaria de Magisterio "Sagrada Familia".

\subsection{Variables e instrumentos}

Las variables relativas a los factores académicos estudiados fueron registradas mediante un cuestionario ad hoc, mientras que para valorar el clima motivacional hacia el deporte se empleó un instrumento diseñado para tal fin. Las variables e instrumentos utilizados se detallan a continuación:

Acceso. Define la vía de acceso a la universidad de los participantes. Esta variable se categoriza en 1=Bachillerato y 2=Formación Profesional (FP).

Nota. Categoriza la nota de acceso a la universidad de los participantes sobre diez. Se codifica en 1=Aprobado, 2=Bien, 3=Notable y 4=Sobresaliente.

Centro. Determina la tipología del centro universitario en el que se encuentra matriculado cada participante. La variable se codifica en 1=Público (Facultad de Ciencias de la Educación de la Universidad de Granada) y 2=Concertado/Privado (Centro de Magisterio "La Inmaculada" y Escuela Universitaria de Magisterio "Sagrada Familia" de Granada).

Beca. Establece si los participantes han recibido beca o algún tipo de ayuda de carácter económico para realizar sus estudios universitarios en el último año.

Clima Motivacional Percibido en el Deporte (PMCSQ-2). La versión original de este instrumento procede de Newton et al. (2000), y fue validado al castellano por González-Cutre, Sicilia y Moreno (2008). El test se compone de 33 ítems, los cuales se puntúan mediante una escala tipo Likert de cinco opciones 
(1=Totalmente en desacuerdo; 5=Totalmente de Acuerdo). Los ítems se estructuran en dos categorías con tres subcategorías para cada una. La dimensión Clima Tarea comprende el Aprendizaje Cooperativo (ítems 11, 21, 31 y 33), el Esfuerzo/Mejora (ítems 1, 8, 14, 16, 20, 25, 28 y 30) y el Papel importante (ítems 4, 5, 10, 19 y 32). La dimensión Clima Ego se constituye por el Castigo por Errores (ítems 2, 7, 9, 15, 18 y 27), el Reconocimiento Desigual (ítems 3, 13, 17, 22, 24, 26 y 29) y la Rivalidad entre Miembros (ítems 6, 12 y 23). En el estudio se obtuvo una consistencia interna de $a=0.79$, la cual fue aceptable.

\subsection{Procedimiento}

En primer lugar se solicitó la colaboración de los participantes a través de una carta informativa elaborada desde el Departamento de Didáctica de la Expresión Musical, Plástica y Corporal de la Universidad de Granada. Posteriormente, se realizó la recogida de los datos en aquellos estudiantes que decidieron participar. Este proceso se realizó en horario lectivo en los centros descritos, sin que ocurriese ningún tipo de incidencia. Asimismo, se contó con la presencia de los investigadores para asegurar una correcta aplicación de los instrumentos descritos. Cabe señalar que se respetó la confidencialidad de todos los participantes y se invalidaron 38 cuestionarios por estar mal cumplimentados.

\subsection{Análisis de los datos}

El análisis estadístico se ha realizado a través del software SPSS 22.0. Para los análisis descriptivos básicos se emplearon frecuencias y medias. El estudio de relaciones entre variables de tipo nominal y de intervalo se realizó a través de $t$ de Student para los factores dicotómicos y Anova de un factor para aquellos con más de dos categorías. La normalidad de los datos se comprobó mediante el test de KolmogorovSmirnov, utilizando la corrección de Lillieforts y la homocedasticidad a través del test de Levene. La fiabilidad interna de los instrumentos empleados fue determinada mediante el alpha de Cronbach, fijando el Índice de Confiabilidad en el 95.5\%.

\section{Resultados}

La relación establecida entre el clima motivacional hacia el deporte y la vía de acceso a la universidad no arrojó diferencias estadísticamente significativas (tabla I).

Tabla I. Clima motivacional según vía de acceso a la universidad

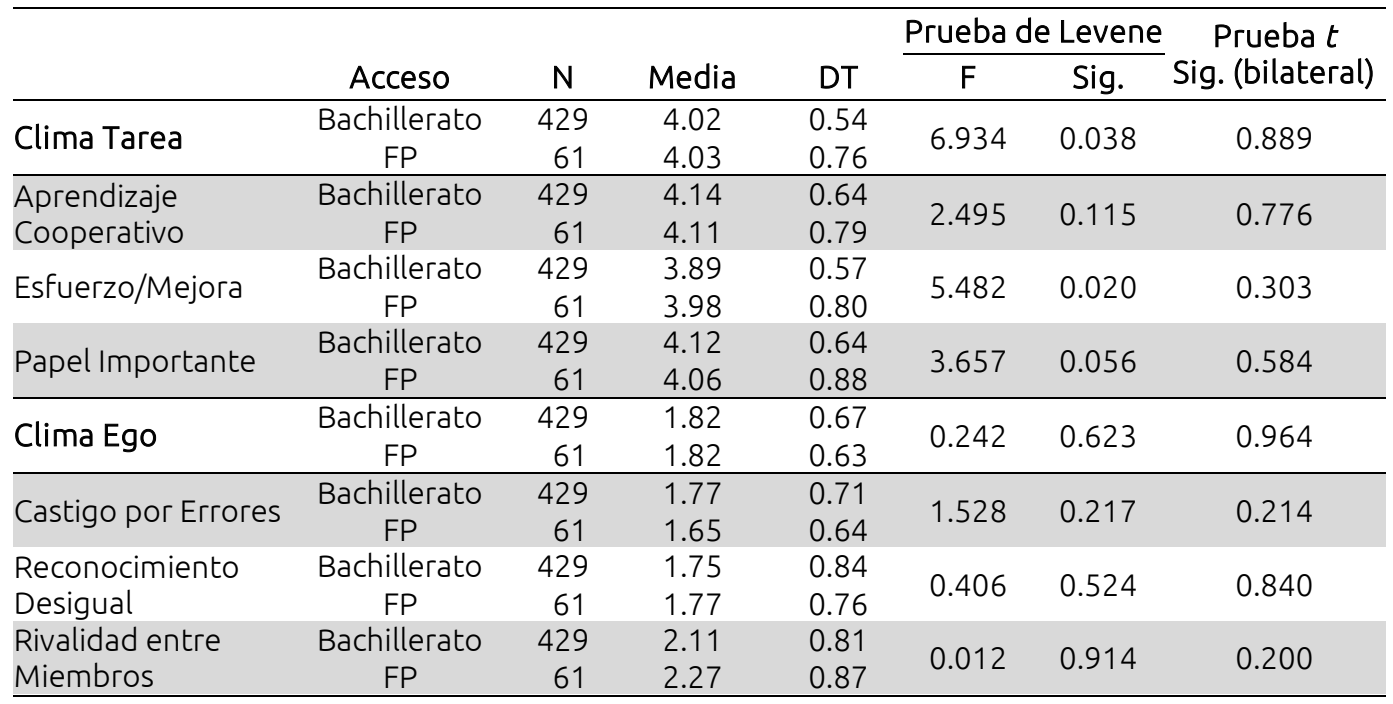

La tabla II muestra el estudio del clima motivacional según la calificación de acceso a la universidad como otro de los factores estudiados en relación con la variable dependiente. En este caso, las puntuaciones 
obtenidas para el $P$ valor revelan únicamente diferencias estadísticas para la categoría Papel Importante $(p=0.045 *)$. Estos valores quedan reflejados en que los estudiantes que acceden a la universidad con una calificación de sobresaliente $(4.32 \pm 0.53)$ obtienen un valor medio superior para esta categoría que los que acceden con notas cualitativas iguales a notable, bien o suficiente $(3.97 \pm 0.70 ; 4.12 \pm 0.68 ; 4.16 \pm 0.66)$, respectivamente.

Tabla II. Clima motivacional según nota de acceso a la universidad

\begin{tabular}{|c|c|c|c|c|c|c|}
\hline & Nota & $\mathrm{N}$ & Media & DT & $\mathrm{F}$ & $p$ \\
\hline \multirow{4}{*}{ Clima Tarea } & Aprobado & 129 & 4.09 & 0.56 & \multirow{4}{*}{1.475} & \multirow{4}{*}{0.220} \\
\hline & Bien & 254 & 4.01 & 0.57 & & \\
\hline & Notable & 84 & 3.93 & 0.60 & & \\
\hline & Sobresaliente & 23 & 4.09 & 0.50 & & \\
\hline \multirow{4}{*}{$\begin{array}{l}\text { Aprendizaje } \\
\text { Cooperativo }\end{array}$} & Aprobado & 129 & 4.19 & 0.65 & \multirow{4}{*}{1.037} & \multirow{4}{*}{0.376} \\
\hline & Bien & 254 & 4.11 & 0.65 & & \\
\hline & Notable & 84 & 4.10 & 0.74 & & \\
\hline & Sobresaliente & 23 & 4.30 & 0.52 & & \\
\hline \multirow{4}{*}{ Esfuerzo/Mejora } & Aprobado & 129 & 4.00 & 0.60 & \multirow{4}{*}{1.655} & \multirow{4}{*}{0.176} \\
\hline & Bien & 254 & 3.89 & 0.61 & & \\
\hline & Notable & 84 & 3.83 & 0.59 & & \\
\hline & Sobresaliente & 23 & 3.84 & 0.59 & & \\
\hline \multirow{4}{*}{ Papel Importante } & Aprobado & 129 & 4.16 & 0.66 & \multirow{4}{*}{2.178} & \multirow{4}{*}{$0.045^{*}$} \\
\hline & Bien & 254 & 4.12 & 0.68 & & \\
\hline & Notable & 84 & 3.97 & 0.70 & & \\
\hline & Sobresaliente & 23 & 4.32 & 0.53 & & \\
\hline \multirow{4}{*}{ Clima Ego } & Aprobado & 129 & 1.79 & 0.66 & \multirow{4}{*}{0.545} & \multirow{4}{*}{0.652} \\
\hline & Bien & 254 & 1.82 & 0.65 & & \\
\hline & Notable & 84 & 1.91 & 0.76 & & \\
\hline & Sobresaliente & 23 & 1.82 & 0.49 & & \\
\hline \multirow{4}{*}{$\begin{array}{l}\text { Castigo por } \\
\text { Errores }\end{array}$} & Aprobado & 129 & 1.72 & 0.67 & \multirow{4}{*}{0.791} & \multirow{4}{*}{0.500} \\
\hline & Bien & 254 & 1.73 & 0.67 & & \\
\hline & Notable & 84 & 1.86 & 0.86 & & \\
\hline & Sobresaliente & 23 & 1.79 & 0.61 & & \\
\hline \multirow{4}{*}{$\begin{array}{l}\text { Reconocimiento } \\
\text { Desigual }\end{array}$} & Aprobado & 129 & 1.76 & 0.82 & \multirow{4}{*}{0.237} & \multirow{4}{*}{0.871} \\
\hline & Bien & 254 & 1.74 & 0.85 & & \\
\hline & Notable & 84 & 1.80 & 0.86 & & \\
\hline & Sobresaliente & 23 & 1.65 & 0.54 & & \\
\hline \multirow{4}{*}{$\begin{array}{l}\text { Rivalidad entre } \\
\text { Miembros }\end{array}$} & Aprobado & 129 & 1.99 & 0.81 & \multirow{4}{*}{2.092} & \multirow{4}{*}{0.100} \\
\hline & Bien & 254 & 2.16 & 0.80 & & \\
\hline & Notable & 84 & 2.24 & 0.89 & & \\
\hline & Sobresaliente & 23 & 2.27 & 0.73 & & \\
\hline
\end{tabular}

El análisis del clima motivacional, según la tipología del centro universitario, arrojó diferencias estadísticamente significativas para la categoría Esfuerzo/Mejora $(p=0.003 *)$ y Reconocimiento Desigual $\left(p=0.035^{*}\right.$ ) (tabla III). Los estadísticos revelan que los universitarios matriculados en centros concertados 0 privados muestran valores medios más elevados en ambas dimensiones. En el caso de la categoría Esfuerzo/Mejora, los estudiantes de centros privados reflejan valores medios más elevados que los de

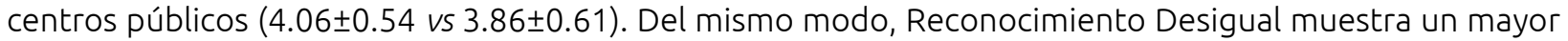
valor medio en el caso de los centros privados con respecto a las universidades públicas (1.92 \pm 0.97 vs 1.70 $\pm 0.78)$. 
Tabla III. Clima motivacional según la tipología del centro universitario

\begin{tabular}{|c|c|c|c|c|c|c|c|}
\hline & \multirow[b]{2}{*}{ Centro } & \multirow[b]{2}{*}{$\mathrm{N}$} & \multirow[b]{2}{*}{ Media } & \multirow[b]{2}{*}{ DT } & \multicolumn{2}{|c|}{ Prueba de Levene } & \multirow{2}{*}{$\begin{array}{c}\text { Prueba } t \\
\text { Sig. (bilateral) }\end{array}$} \\
\hline & & & & & $\mathrm{F}$ & Sig. & \\
\hline \multirow{2}{*}{ Clima Tarea } & Público & 378 & 3.99 & 0.58 & \multirow{2}{*}{0.987} & \multirow{2}{*}{0.321} & \multirow{2}{*}{0.057} \\
\hline & Privado & 112 & 4.11 & 0.52 & & & \\
\hline \multirow{2}{*}{$\begin{array}{l}\text { Aprendizaje } \\
\text { Cooperativo }\end{array}$} & Público & 378 & 4.12 & 0.67 & \multirow{2}{*}{0.972} & \multirow{2}{*}{0.325} & \multirow{2}{*}{0.247} \\
\hline & Privado & 112 & 4.21 & 0.62 & & & \\
\hline \multirow{2}{*}{ Esfuerzo/Mejora } & Público & 378 & 3.86 & 0.61 & \multirow{2}{*}{0.952} & \multirow{2}{*}{0.330} & \multirow{2}{*}{$0.003^{*}$} \\
\hline & Privado & 112 & 4.06 & 0.54 & & & \\
\hline \multirow{2}{*}{ Papel Importante } & Público & 378 & 4.11 & 0.69 & \multirow{2}{*}{0.002} & \multirow{2}{*}{0.963} & \multirow[b]{2}{*}{0.789} \\
\hline & Privado & 112 & 4.13 & 0.63 & & & \\
\hline \multirow{2}{*}{ Clima Ego } & Público & 378 & 1.81 & 0.65 & \multirow{2}{*}{0.548} & \multirow{2}{*}{0.459} & \multirow{2}{*}{0.441} \\
\hline & Privado & 112 & 1.87 & 0.71 & & & \\
\hline \multirow{2}{*}{$\begin{array}{l}\text { Castigo } \\
\text { por Errores }\end{array}$} & Público & 378 & 1.78 & 0.72 & \multirow{2}{*}{0.069} & \multirow{2}{*}{0.792} & \multirow{2}{*}{0.19} \\
\hline & Privado & 112 & 1.68 & 0.65 & & & \\
\hline \multirow{2}{*}{$\begin{array}{l}\text { Reconocimiento } \\
\text { Desigual }\end{array}$} & Público & 378 & 1.70 & 0.78 & \multirow{2}{*}{1.291} & \multirow{2}{*}{0.001} & \multirow{2}{*}{$0.035^{*}$} \\
\hline & Privado & 112 & 1.92 & 0.97 & & & \\
\hline \multirow{2}{*}{$\begin{array}{l}\text { Rivalidad entre } \\
\text { Miembros }\end{array}$} & Público & 378 & 2.13 & 0.80 & \multirow{2}{*}{2.470} & 0117 & 097 \\
\hline & Privado & 112 & 2.13 & 0.89 & & & \\
\hline
\end{tabular}

La concesión de beca o cuantía económica para cursar estudios universitarios fue el último factor académico estudiado en relación con el clima motivacional percibido (ver tabla IV). Tal y como revela la significatividad bilateral de la prueba $t$ para muestras independientes no se obtuvieron diferencias estadística en este análisis.

Tabla IV. Clima motivacional según concesión de beca

\begin{tabular}{|c|c|c|c|c|c|c|c|}
\hline & & & & & & $\begin{array}{l}\text { ba } \\
\text { ene }\end{array}$ & Prueba $t$ \\
\hline Categoría & Beca & $\mathrm{N}$ & Media & DT & $\mathrm{F}$ & Sig. & \\
\hline & Sí & 240 & 4.05 & 0.51 & & & \\
\hline Clima laгеа & No & 250 & 3.99 & 0.62 & 4.912 & 0.027 & 0.319 \\
\hline Aprendizaje & Sí & 240 & 4.15 & 0.63 & & & \\
\hline Cooperativo & No & 250 & 4.13 & 0.69 & $1 . \angle U L$ & 0.274 & 0.121 \\
\hline Esfuerzo/Mejora & Sí & 240 & 3.93 & 0.53 & 8292 & 0004 & 0265 \\
\hline 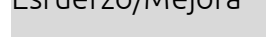 & No & 250 & 3.87 & 0.67 & 0.232 & 0.004 &  \\
\hline Papel Importante & Sí & 240 & 4.15 & 0.65 & 0774 & 0380 & 0320 \\
\hline & No & 250 & 4.08 & 0.70 & 0.174 & 0.380 & \\
\hline & Sí & 240 & 1.80 & 0.69 & & & \\
\hline Clima Ego & No & 250 & 1.85 & 0.64 & 2.853 & 0.092 & 0.466 \\
\hline Castigo por & Sí & 240 & 1.74 & 0.72 & & & \\
\hline Errores & No & 250 & 1.76 & 0.70 & 1.258 & 0.263 & 0.114 \\
\hline Reconocimiento & Sí & 240 & 1.73 & 0.87 & 1330 & 0.249 & 0.542 \\
\hline Desigual & No & 250 & 1.78 & 0.79 & & 0.249 & 0.342 \\
\hline Rivalidad entre & Sí & 240 & 2.09 & 0.84 & 0.707 & 0.401 & 0.274 \\
\hline Miembros & No & 250 & 2.17 & 0.80 & 0.101 & 0.401 & 0.274 \\
\hline
\end{tabular}

\section{Discusión y conclusiones}

El estudio realizado tuvo como objetivo relacionar el clima motivacional percibido hacia el deporte con diversos factores de tipo académico para conocer las motivaciones hacia la práctica deportiva de los futuros docentes, así como la influencia que pueden ejercer elementos académicos-como el rendimiento académico o la tipología del centro universitario. Algunos estudios de características similares fueron realizados por Atkins, Johnson, Force y Petrie (2015), Méndez-Giménez et al. (2013), y Salmerón, 
Gutiérrez, Salmerón y Rodríguez (2011).

Los descriptivos revelaron que la vía de acceso a la universidad o la concesión de beca para estudiar no se relacionaban con las orientaciones de meta en la práctica deportiva. Breuer, Hallman, Wicker y Feiler (2010), y Martínez-Mendoza y Martínez-Mendoza (2013) concretan la concesión de cuantías económicas para estudiar cómo una motivación extrínseca puede influir en el desempeño académico y deportivo. Sin embargo, este estudio consideró la concesión de becas para cursar estudios superiores y no para la realización de actividades físico-deportivas concretas, motivo por el cual no se obtuvieron diferencias estadísticas en relación con el clima motivacional.

No obstante, se pudo apreciar que la tipología del centro universitario en el que se realizan los estudios superiores sí se asociaba con las metas de logro hacia el deporte de los estudiantes. Estas diferencias estadísticas se evidencian en que aquellos participantes matriculados en universidades privadas eran los que más se esforzaban para mejorar en la práctica deportiva y los que más perseguían el reconocimiento desigual. En un estudio similar realizado con un grupo de adolescentes, Coterón, Franco, Pérez-Tejero y Sampedro (2013) obtuvieron que los estudiantes matriculados en centros privados se orientaban en mayor medida al Clima Ego, lo que puede deberse a la educación más elitista y personalizada de este tipo de centros, así como la promoción de la competencia a través de primas y motivaciones extrínsecas a los alumnos más habilidosos (Agasisti y Ricca, 2015; Méndez-Giménez et al., 2013). Por otro lado, el presente estudio se realizó en estudiantes universitarios, etapa educativa en la que existe una mayor competencia por lograr mejores calificaciones y salidas profesionales (Martínez-Martínez, 2013), lo que podría influir indirectamente en su clima motivacional hacia el deporte.

Sobre las calificaciones de acceso a la universidad se obtuvo que los estudiantes que preferían tener un papel protagonista en la práctica deportiva eran los que accedieron con mayor calificación. González y Portolés (2014) destacan la importancia de la motivación intrínseca en el aula con el fin de mejorar el rendimiento académico, justificando estos resultados. De hecho, Richardson, Abraham y Bond (2012) establecen que aquellos estudiantes a los que les gusta implicarse en una tarea y en el proceso para conseguir unos objetivos, son los que presentan conductas más autodeterminadas relacionadas con motivaciones intrínsecas, que por regla general son las que se asocian con mejor rendimiento académico (Ardoy et al., 2013; Camacho-Miñano y del Campo, 2015; Salmerón et al., 2011).

Este trabajo complementa una línea de investigación de gran interés, como es el estudio del clima motivacional hacia el deporte en el ámbito educativo, y da un paso más al analizar este componente motivacional en la etapa universitaria en relación con múltiples factores académicos, destacando los beneficios de la práctica deportiva a nivel cognitivo. Sin embargo, es necesario mencionar las principales limitaciones del estudio: la primera se relaciona con el diseño metodológico (de tipo descriptivo y corte transversal), diseño que permite observar asociaciones entre variables, pero no confeccionar relaciones de causa-efecto, algo que sí permitiría un diseño longitudinal. Otras limitaciones se derivan de las variables utilizadas; el rendimiento académico fue valorado mediante un cuestionario ad hoc y no mediante un instrumento validado (como se hizo en el caso del clima motivacional), lo que podría ofrecer resultados más ajustados a la realidad. Sin embargo, literatura reciente define a las calificaciones como un buen indicador del rendimiento académico, aunque no del desempeño académico.

Entre las conclusiones, se revela cómo algunos factores de índole académica pueden asociarse con la motivación hacia la práctica deportiva, dado que los universitarios con mayor rendimiento académico mostraban motivaciones más autodeterminadas e intrínsecas, como tener un papel importante en la práctica de deporte. Asimismo, se observó que los estudiantes matriculados en centros privados perseguían en mayor medida el reconocimiento desigual y el esfuerzo para la mejora, lo que revela cómo el estilo de enseñanza docente puede asociarse con la configuración de climas motivacionales concretos hacia el deporte. 


\section{Referencias}

Agasisti, T. y Ricca, L. (2015). Comparing the Efficiency of Italian Public and Private Universities (20072011): An Empirical Analysis. Italian Economic Journal, 2(1), 57-89. doi:10.1007/s40797-015-0022-7

Alfonso, R. M. (2016). Relación entre la actividad física extraescolar y el rendimiento académico en alumnos de Educación Secundaria. Sportis, 11(2), 177-187.

Almagro, B., Sáenz-López, P. y Moreno-Murcia, J. A. (2012). Perfiles motivacionales de deportistas adolescentes españoles. Revista de Psicología del Deporte, 21(2), 223-231.

Aránguiz, C. y Rivera, P. (2012). Competencias transversales en los planes de estudio de las titulaciones de grado: Los esfuerzos de Bolonia en calidad universitaria. Revista Entornos, 25(2), 105-117.

Ardoy, D. N., Fernández-Rodríguez, J. M., Jiménez-Pavón, D., Castillo, R., Ruiz, J. R. y Ortega, F. B. (2013). A Physical Education trial improves adolescents' cognitive performance and academic achievement: the EDUFIT study. Scandinavian Journal of Medicine \& Science in Sports, 24(1), 52-61. doi:10.1111/sms.12093

Atkins, M., Johnson, D., Force, E. y Petrie, T. (2015). Peers, parents and coaches, oh my! The relation of the motivational climate to boys' intention to continue in sport. Psychology of Sport and Exercise, 16(3), 170180. doi:10.1016/j.psychsport.2014.10.008

Ayan, C., Besada, A., Cancelas, J. M. y Martínez, L. (2015). Is there any link between aerobic performance and academic achievement in preschool children? Findings from a pilot study. Journal of Sport and Health Research, 7(1), 11-18.

Camacho-Miñano, M. M. y Del Campo, C. (2015). Impacto de la motivación intrínseca en el rendimiento académico a través de trabajos voluntarios: Un análisis empírico. Revista Complutense de Educación, 26(1), 67-80.

Castillo, I., Balaguer, I. y Duda, J. L. (2000). Las orientaciones de meta y los motivos de práctica deportiva en los jóvenes deportistas valencianos escolarizados. Revista de Psicología del Deporte, 9(2), 37-50.

Castro-Sánchez, M., Zurita-Ortega, F., Chacón-Cuberos, R., Martínez-Martínez, A., Espejo-Garcés, T. y Álvaro-González, J. I. (2015). Sustancias nocivas y clima motivacional en relación a la práctica de actividad física. Health and Addictions, 15(2), 115-126. doi:10.21134/haaj.v15i2.244

Coterón, J., Franco, E., Pérez-Tejero, J. y Sampedro, J. (2013). Clima motivacional, competencia percibida, compromiso y ansiedad en Educación Física. Diferencias en función de la obligatoriedad de la enseñanza. Revista de Psicología del Deporte, 22(1), 151-157.

Cuevas, R., García-Calvo, T. y Contreras, O. (2013). Perfiles motivacionales en Educación Física: una aproximación desde la teoría de las Metas de Logro 2x2. Anales de Psicología, 29(3), 685-692. doi:10.6018/analesps.29.3.175821

Deci, E. L. y Ryan, R. M. (2000). The "what" and "why" of goal pursuits: Human needs and the selfdetermination of behaviour. Psychological Enquiry, 11(4), 227-268.

Elias, M. y Daza, L. (2014). Sistema de becas y equidad participativa en la universidad. Revista de la Asociación de Sociología de la Educación, 7(1), 233-251.

García-Calvo, T., Sánchez, P. A., Leo, F. M., Sánchez, D. y Amado, D. (2011). Incidencia de la Teoría de Autodeterminación sobre la persistencia deportiva. RICYDE, 25(7), 266-276.

doi:10.5232/ricyde2011.02502 
González, J. y Portolés, A. (2014). Actividad física extraescolar: relaciones con la motivación educativa, rendimiento académico y conductas asociadas a la salud. Revista Iberoamericana de Psicología del Ejercicio y el Deporte, 9(1), 51-65.

González-Cutre, D., Sicilia, A. y Moreno, J. A. (2008). Modelo cognitivo-social de la motivación de logro en educación física. Psicothema, 20(4), 642-651.

Hanus, M. y Fox, J. (2015). Assessing the effects of gamification in the classroom: A longitudinal study on intrinsic motivation, social comparison, satisfacction, effor, and academic performance. Computers \& Education, 80(1), 152-161. doi:10.1016/j.compedu.2014.08.019

Jaakkola, T., Ntoumanis, N. y Liukkonen, J. (2016). Motivational climate, goal orientation, perceived sport ability, and enjoyment within Finnish junior ice hockey players. Scandinavian Journal of Medicine \& Science in Sport, 26(1), 109-115. doi:10.1111/sms.12410

Kusurkar, R. A., Ten Cate, J., Vos, C. M., Westers, P. y Croiset, G. (2013). How motivation affects academic performance: a structural equation modelling analysis. Advances in Health Sciences Education, 18(1), 57-69. doi:10.1007/s10459-012-9354-3

Lisha, N. E. y Sussman, S. (2010). Relationship of high school and college sports participation with alcohol, tobacco, and illicit drug use: A review. Addictive Behaviors, 35(5), 399-407.

doi:10.1016/j.addbeh.2009.12.032

Marcos, J., Orquín, F. J., Belando, N. y Moreno-Murcia, J. A. (2014). Motivación autodeterminada en adultos mayores practicantes de ejercicio físico. Cuadernos de Psicología del Deporte, 14(3), 149-156.

Martínez, A., Castro, M., Zurita, F. y Lucena, M. (2015). La elección de estudios superiores universitarios en función de la modalidad de estudios, la nota media y el género. Magister, 27(1), 18-25.

doi:10.1016/j.magis.2015.06.001

Martínez-Martínez, A. (2013). La orientación como actividad educativa y vocacional en los itinerarios curriculares del alumnado de Bachiller y Formación Profesional y su inclusión en el mercado laboral. Tesis Doctoral. Universidad de Granada, España.

Martínez-Mendoza, E. y Martínez-Mendoza, R. (2013). Análisis del efecto de las becas económicas en el rendimiento escolar en una institución de nivel medio superior. Investigación y Ciencia, 59(1), 41-47.

Méndez-Giménez, A., Fernández-Río, J. y Cecchini-Estrada, J. A. (2014). Análisis de un modelo multiteórico de metas de logro, metas de amistad y autodeterminación en educación física. Estudios de Psicología, 33(3), 325-336.

Méndez-Giménez, A., Fernández-Río, J., Cecchini-Estrada, J. A. y González, C. (2013). Perfiles motivacionales y sus consecuencias en educación física. Un estudio complementario de metas de logro $2 \times 2$ y autodeterminación. Revista de Psicología del Deporte, 22(1), 29-38.

Newton, M., Duda, J. L. y Yin, Z. (2000). Examination of the psychometric properties of the Perceived Motivational Climate in Sport Questionnaire-2 in a sample of female athletes. Journal of Sports Sciences, 18(4), 275-290.

Renault, G., Cortada, N. y Castro, A. (2014). Factores que intervienen en el rendimiento académico de los estudiantes de psicología y psicopedagogía. Signos Universitarios, 27(43), 27-34.

Richardson, M., Abraham, C. y Bond, R. (2012). Psychological correlates of university students academic performance: a systematic review and meta-analysis. Psychological Bulletin, 138(2), 353-387.

doi:10.1037/a0026838 
Salmerón, H., Gutiérrez, C., Salmerón, P. y Rodríguez, S. (2011). Metas de logro, estrategias de regulación y rendimiento académico en diferentes estudios universitarios. Revista de Investigación Educativa, 29(2), 467-486. 